\title{
Energy level determination in bulk heterojunction systems using photoemission yield spectroscopy: case of P3HT:PCBM
}

\author{
Raitis Grzibovskis*, Aivars Vembris
}

Institute of Solid State Physics, University of Latvia, 8 Kengaraga Street, Riga, LV-1063, Latvia

E-mail: raitis.g@cfi.lu.lv*; aivars.vembris@cfi.lu.lv

Phone number: +37167260787

Raitis Grzibovskis ORCID ID: 0000-0001-5863-2295

Aivars Vembris ORCID ID: 0000-0002-9346-946X

\begin{abstract}
:
Ultraviolet photoelectron spectroscopy (UPS) is commonly used method for energy level determination using planar heterojunction samples in either metal/organic or organic/organic systems. Only some attempts have been made in bulk heterojunction system studies. Photoemission yield spectroscopy (PYS) could be applied as a method for organic compound- organic compound interface studies in bulk heterojunction samples. Contrary to the UPS, PYS method does not require ultra-high vacuum which simplifies experiment setup. Also, scanning depth of PYS is in the range of tens of nanometers which allows studying deeper layers of the sample instead of only surface layer.

In this work poly(3-hexylthiophene-2,5-diyl) (P3HT) and [6,6]-phenyl C61 butyric acid methyl ester (PCBM) bulk heterojunction thin films were studied as a model system. A mass ratio between P3HT and PCBM in the system was varied from 1:0 to 1:50. Ionization energy dependence on this ratio was studied using two methods- UPS and PYS. To study the influence of sample morphology on the PYS measurements and obtainable results, phase separated and homogeneously distributed samples were prepared for analyses. P3HT ionization energy shift of $0.40 \mathrm{eV}$ was observed in the samples made from chloroform solution. Experiments showed the need for a low degree of phase separation between P3HT and PCBM to observe P3HT ionization energy shift using PYS. On the contrary, no ionization energy shift of P3HT was observed in the UPS measurements for the same systems.
\end{abstract}


Keywords: photoemission yield spectroscopy, ultraviolet photoelectron spectroscopy, interface, ionization energy, organic materials, thin films

\section{INTRODUCTION}

Devices made of organic materials often consist of several layers, such as electrodes, electron/hole transport layers, and an active layer. Each of these layers is only up to 100nm thick [1-5]. At this thickness, various surface and interface effects become more pronounced. Energy level compatibility at the organic compound-organic compound (OC-OC) and the electrode-organic compound interface can greatly influence the performance of the devices such as organic light emitting diodes (OLEDs) $[6,7]$ and organic photovoltaic (OPV) cells [8-10]. At small film thickness, the ionization energy and electron affinity level shift can be observed due to the Fermi level alignment between two different materials $[11,12]$. Thereby, energy level values obtained from bulky layers cannot be used anymore. Therefore energy level shift at the both OC-OC and the metal-organic interface is being actively studied [12-16].

Ultraviolet photoelectron spectroscopy (UPS) is a common method for molecule ionization energy determination in thin films [17-19]. This method can also be used for the study of the molecule ionization energy shifts at the OC-OC interfaces $[14,20]$. Usually, ionization energy dependence on the layer thickness in planar heterojunction samples is studied. Few attempts have been made to investigate the molecule ionization energy in the bulk heterojunction samples obtained from the solution by the UPS method [21, 22]. Tsoi et al. [22] have investigated pure poly(3-hexylthiophene2,5-diyl) (P3HT) and [6,6]-phenyl C61 butyric acid methyl ester (PCBM) films and their blends. Their experimental results showed that there is no P3HT energy level difference between neat and blend films and there is no PCBM contribution on the energy level alignment. Authors explain it with the almost pure P3HT top layer of the blend. UPS sensitivity is only 2-3 nm thickness which means that they have measured mostly P3HT. Ze-Lei Guan et al. [23] addresses the same issue. The problem was solved by lifting off the film from the substrate. In this way, authors obtained the surface that was rich in both- donor (P3HT) and acceptor (PCBM) compound- which is a better 
representation of the P3HT:PCBM blend. But they had to deal with the substrate influence on the “once-buried surface" energy levels.

Recently, X-ray photoelectron spectroscopy (XPS) has been used as a method for OC-OC interface studies in blend systems. A constant energetic separation between core levels and ionization energy was assumed to obtain ionization energy shift in the blend. Such approach was applied for monolayer and multilayer blend systems of pentacene and phthalocyanine derivatives [24] as well as for P3HT/ DFPyC60 film [25]. In the second case, oxygen influence on energy level shift was investigated. Still, XPS method did not show any energy level shift between compounds in the blend (P3HT/ DFPyC60) without oxygen and separate thin films without oxygen.

Photoemission yield spectroscopy (PYS) could be used as an alternative method for OC-OC interface studies. The method is based on the photoemission yield dependence on photon energy $(Y(h v))$ [26-28]. It offers a possibility to make measurements in the air [28], however more precise results could be obtained in a vacuum $\left(\sim 10^{-5} \mathrm{mBar}\right)$. Comparing to the UPS measurements which can be performed only in ultra-high vacuum $\left(<10^{-9} \mathrm{mBar}\right)$, PYS is less complex and less expensive method. One more and the most important advantage of the PYS method is it's scanning depth, which is in the range of tens of nanometers due to the relatively low photon energy (in the range of $4-8 \mathrm{eV}$ ) [29]. In comparison, the scanning depth of the UPS is only up to $2-3 \mathrm{~nm}$.

In this work, we have evaluated the applicability and limitations of PYS for OC-OC interface studies in bulk heterojunction samples. Similarly, as in the case of UPS, PYS has been mostly used in the ionization energy measurements of metal and pure organic compound films [27, 30]. There are only some research done in metal/organic compound [26] or OC-OC interface studies [31, 32].

P3HT:PCBM bulk heterojunction thin films were chosen as a model system in this study. P3HT ionization energy shift was measured at various mass ratios between P3HT and PCBM. The mass ratio in the system was changed to obtain similar conditions as in planar samples where usually film thickness is varied. P3HT:PCBM system was chosen due to a large amount of research on P3HT and PCBM thin films and their energy level values. Such approach gives a possibility to evaluate the validity of our results and the method itself. Thus far only pure films of P3HT [33] and PCBM [31] have been studied using PYS. To our best knowledge this method has not been applied to P3HT:PCBM as well as any other bulk heterojunction system thus far. 
To study the influence of the sample morphology on the PYS measurements and obtainable results, phase separated and homogeneously distributed samples were prepared for analyses. Such systems were obtained by using two solvents in the sample preparation: chlorobenzene and chloroform.

Samples made from chloroform were additionally investigated by UPS for comparison with its alternative - PYS method.

\section{EXPERIMENTAL}

\subsection{Studied compounds}

In this work bulk heterojunction samples made from two well-known OPV materials- hole transport polymer P3HT (regioregular, 99.995\%, Sigma Aldrich) and electron transport fullerene derivative PCBM ( $>99.5 \%$, Sigma Aldrich) - were studied. Molecule structure of the studied compounds is shown in Figure 1.
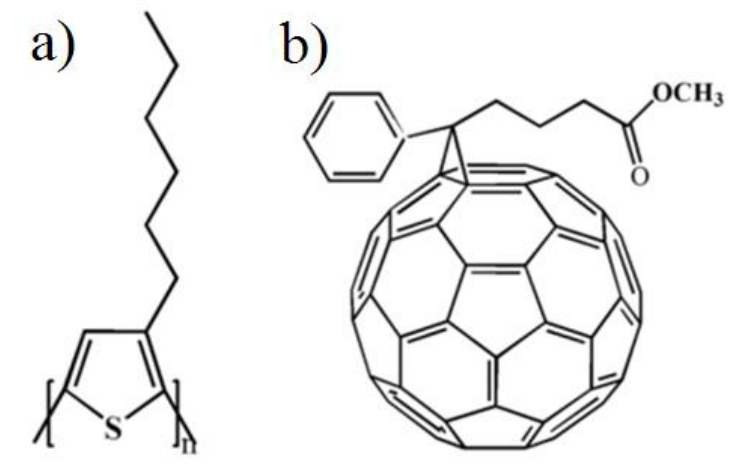

Fig.1 Chemical structure of the studied compounds: a) P3HT and b) PCBM

\subsection{Sample preparation}

Two series of the bulk heterojunction samples with structure ITO/P3HT:PCBM were prepared on ITO covered glass substrates (Präzisions Glas \& Optik GmbH). In one case as a solvent, we used chlorobenzene while in the other series chloroform was applied. As the boiling point of chloroform is relatively low $\left(61^{\circ} \mathrm{C}\right)$ it evaporates fast. This rapid evaporation ensures low phase separation of 
P3HT and PCBM within the bulk of the samples. It leads to the decreased distance between two PCBM molecules as PCBM concentration increases in the system. It would be similar to a decrease of P3HT layer thickness for multilayer samples. On the contrary, the chlorobenzene evaporates slowly as the boiling point of it is relatively high $\left(131^{\circ} \mathrm{C}\right)$. This slow evaporation ensures that phase separation between P3HT and PCBM can occur and organic compounds are no more homogenously distributed within the bulk of the sample. Therefore chlorobenzene [34-38] or dichlorobenzene (boiling point of $180^{\circ} \mathrm{C}$ ) $[39,40]$ is often used as a solvent in the production of highly efficient P3HT:PCBM solar cells.

Solutions with the concentration of $30 \mathrm{mg} \mathrm{ml}^{-1}$ were prepared. In both series the samples with the mass ratio between P3HT and PCBM of 1:0 (pure P3HT layer), 1:1, 1:3, 1:5, 1:10, 1:20, and 1:50 were made. The solution was spin-coated using Laurell WS-650Sx-GNPP/Lite spin-coater on the ITO covered glass. Spin-coating parameters were kept unchanged: spinning speed- $400 \mathrm{rpm}$, acceleration- $400 \mathrm{rpm} \mathrm{s}^{-1}$, spinning time- $40 \mathrm{~s}$. The samples were dried on a hot plate. The samples made from the chloroform solution were heated at $70^{\circ} \mathrm{C}$ for 20 minutes. The samples made from the chlorobenzene solution were dried at $120^{\circ} \mathrm{C}$ for 20 minutes and then additional 10 minutes at $150^{\circ} \mathrm{C}$. The thickness of all obtained samples was about 300nm. This thickness was enough to exclude any influence of the ITO/organic compound interface.

The sample morphology was studied by scanning electron microscope Tescan Lyra FE-FIB-SEM.

\subsection{Photoemission measurements}

Photoemission yield spectroscopy measurements were done in a vacuum (pressure of about $10^{-5}$ mBar) using a self-built measurement system. ENERGETIQ Laser-Driven Light Source (LDLS EQ99) was used as a source of ultraviolet radiation. The spectral range of the measurements was between $4 \mathrm{eV}$ and $6.5 \mathrm{eV}$. Incident photon energy was changed by diffraction grating monochromator MYM-1 with the step of $0.05 \mathrm{eV}$. The samples were irradiated through the quartz window of the vacuum chamber. The distance between the sample and electrons collecting electrode was about $2 \mathrm{~cm}$. Keithley 617 electrometer was used as the equipment for electrical current measurements as well as a voltage source. Applying a voltage of $50 \mathrm{~V}$ between the sample and the electrode did not 
change the form of the obtained spectrum but improved signal to noise ratio by one order of magnitude. The set-up of the measurement system is published elsewhere [41].

Ultraviolet photoelectron spectroscopy measurements were carried out using PHI VersaProbe III. He I lamp with a photon energy of $21.2 \mathrm{eV}$ was used as an excitation source. Ionization energy $(I)$ of the material with the respect to the vacuum level is calculated as

$$
I=h v-\left(E_{S E C O}-E_{L B E}\right)
$$

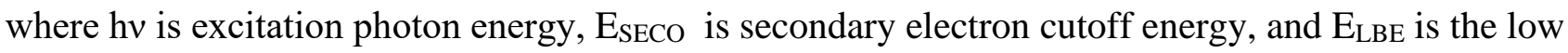
binding energy onset.

\section{RESULTS AND DISCUSSION}

\subsection{Sample morphology}

A pure P3HT film made from chlorobenzene was smooth without any structures (not shown here). Other samples made from chlorobenzene solution were different. As the PCBM mass in the sample increased, PCBM crystallites could be observed on the surface of the sample (Fig.2 $\mathrm{c}$ and d). When the P3HT:PCBM mass ratio was 1:3, the PCBM crystallites were around $20 \mu \mathrm{m}$ long (Fig.2 c). When P3HT:PCBM mass ratio was changed to 1:10, PCBM crystallites covered even more of the surface and their size increased to $50-60 \mu \mathrm{m}$. Formed crystallites were no more linear but created branch-like structures (see Fig.2 d). It is in contradiction with few previous papers which state that in P3HT:PCBM samples the surface layer is almost pure P3HT due to the lower surface energy of P3HT [21, 22, 42]. Nevertheless, there is a report [43] where result similar to our case was shown. Most likely preparation conditions like annealing temperature could influence PCBM aggregation on the surface. High PCBM concentration and the high annealing temperature can lead to extensive PCBM aggregation on the surface of the sample. This leaves regions around fullerene crystallites to be PCBM-depleted. In Fig.2 c clear contrast between P3HT and PCBM regions can be observed.

Samples made from chloroform were smooth and without visible structures (Fig.2 a and b). Within the chosen observation range (tens of micrometers) the films seemed to be amorphous where P3HT 
and PCBM are homogeneously distributed in the bulk of the sample. Even when the mass ratio of P3HT:PCBM was 1:10, no PCBM crystallites could be observed (Fig.2 b). Similarly, samples with higher PCBM concentration were still smooth and without any fullerene crystallites. Fast evaporation of chloroform and relatively low annealing temperature $\left(70^{\circ} \mathrm{C}\right)$ precludes extensive phase separation between P3HT and PCBM. It has been reported that P3HT:PCBM films made from chloroform solution have the surface roughness of around $1 \mathrm{~nm}$ and aggregates in the range of a couple of tens of nanometers [44].

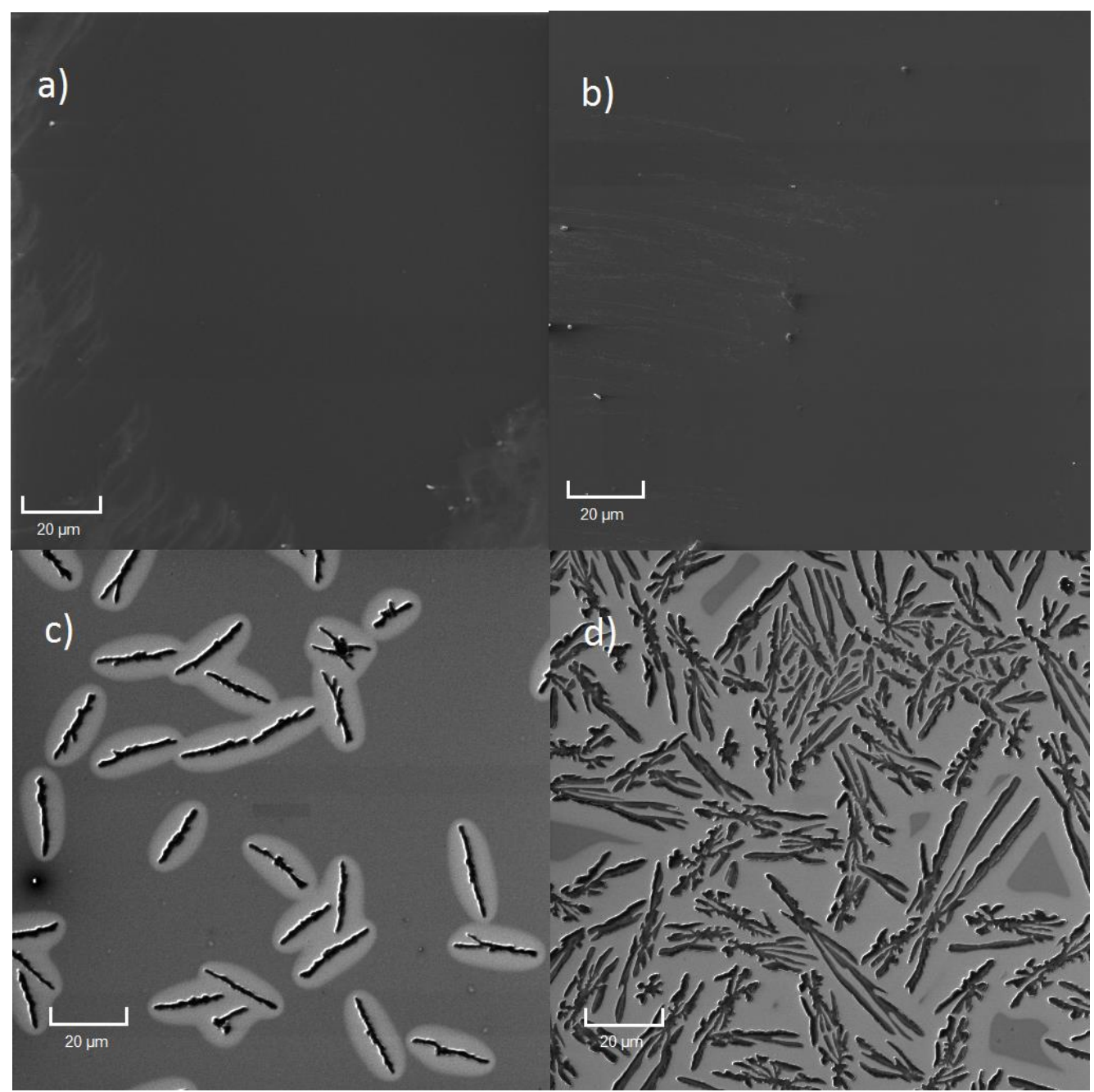

Fig.2 SEM images of a) P3HT sample made from the chloroform solution; b) P3HT:PCBM (1:10) sample made from the chloroform; c) P3HT:PCBM (1:3) sample made from the chlorobenzene solution; d) P3HT:PCBM (1:10) sample made from the chlorobenzene solution. Magnification in all the cases is 1000x 


\subsection{Ionization energy measurements}

Photoemission yield $\mathrm{Y}(h v)$ can be calculated as

$$
Y(h v)=I(h v) / P(h v)
$$

where $\mathrm{I}(h v)$ is the number of emitted electrons and $\mathrm{P}(h v)$ is the number of incident photons with the energy of $h v$. In threshold region, a relation between photoemission yield $\mathrm{Y}(h v)$ and ionization energy $\mathrm{E}_{\text {ioniz }}$ can be expressed as a power law

$$
Y(h v)=\alpha\left(h v-E_{\text {ioniz }}\right)^{n}
$$

where $\alpha$ is constant showing amplitude of the signal and $n=1 \ldots 3$ depending on studied materials [45]. $n=2$ is used in the case of metals $[30,46]$, while $n=2.5$ or $n=3$ is used in the case of semiconductors $[14,26,27]$. In this work, we have used $n=2.5$ as it gave better approximation than $n=3$.

\subsubsection{Bulk heterojunction samples made from chlorobenzene}

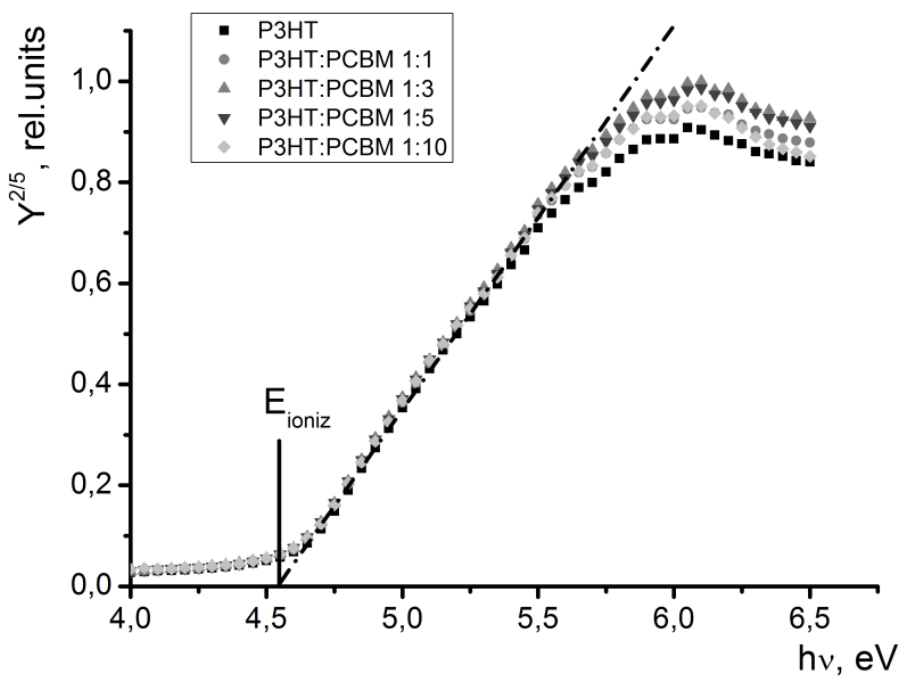


Fig.3 Photoemission yield spectra of bulk heterojunction samples depending on P3HT:PCBM mass ratio. Samples made from chlorobenzene solution

To obtain ionization energy value $E_{\text {ioniz }}$ from photoemission yield measurements, $\mathrm{Y}^{2 / 5}(\mathrm{~h} v)$ is calculated and plotted depending on photon energy. Then the linear part of $\mathrm{Y}^{2 / 5}(\mathrm{hv})$ curve is extrapolated till $\mathrm{Y}^{2 / 5}(\mathrm{~h} v)=0$. Cross section point with the $\mathrm{x}$-axis is considered to be the ionization energy of the studied compound (Fig.3).

For all the samples made from chlorobenzene solution, the photoelectron emission signal was obtained only from P3HT with an insignificant influence of PCBM (see Fig.3). Even when the mass ratio of P3HT:PCBM was 1:10, no notable signal coming from PCBM was observed. This could be explained by two effects. First, in our previous research, we observed that signal created by pure PCBM film at the photon energy of $6.50 \mathrm{eV}$ is at least one order of magnitude lower than signal created by pure P3HT film [32]. It might be related to the difference in hole mobility of the studied compounds- $\mathrm{P} 3 \mathrm{HT}$ is hole conducting material while PCBM is electron conductor. Second, the ionization energy of PCBM is close to $6.00 \mathrm{eV}$ [32], but the ionization energy of P3HT is close to $4.50 \mathrm{eV}$ (see Fig.3). It means that UV radiation with photon energy just above $6.00 \mathrm{eV}$ can ionize only small part of PCBM molecules while it is almost $1.50 \mathrm{eV}$ over the ionization threshold energy of P3HT. Thus polymer molecules can be easily ionized. The combination of these two effects can "hide" any PCBM influence on the photoemission signal.

In all the cases obtained ionization energy value of P3HT was the same $\left(\mathrm{I}_{\mathrm{P} 3 \mathrm{HT}}=4.54 \pm 0.03 \mathrm{eV}\right)$ which matches the energy value of the pure P3HT sample. The signal of measurements is a sum of two separate signals [26, 47], in our case the signal created by the molecules at the interface and the signal created by the molecules within the region of pure P3HT. It seems that in this case most of the signal comes from the bulky part of the P3HT and not the OC-OC interface. It means that phaseseparated samples made from chlorobenzene even at high PCBM concentrations cannot be used for P3HT:PCBM interface studies due to the small amount of the molecules at the P3HT/PCBM interface comparing to the molecules at regions of pure P3HT. To observe interface effects, we need as many P3HT molecules as possible at the interface with PCBM. 


\subsubsection{Bulk heterojunction samples made from chloroform}

To increase the number of molecules at the OC-OC interface, series of the samples were made from chloroform solution. As discussed in section 2.2, the fast evaporation of chloroform precludes extensive aggregation of P3HT and PCBM within the bulk of the sample.

Photoemission yield spectrum and the ionization energy of pure P3HT sample made from chloroform was the same as for the sample made from chlorobenzene (see Fig.4):

$\mathrm{I}_{\mathrm{P} 3 \mathrm{HT}}=4.54 \pm 0.03 \mathrm{eV}$. It means that there is no distinguishable influence of used solvents and thermal annealing on the energy levels of P3HT.

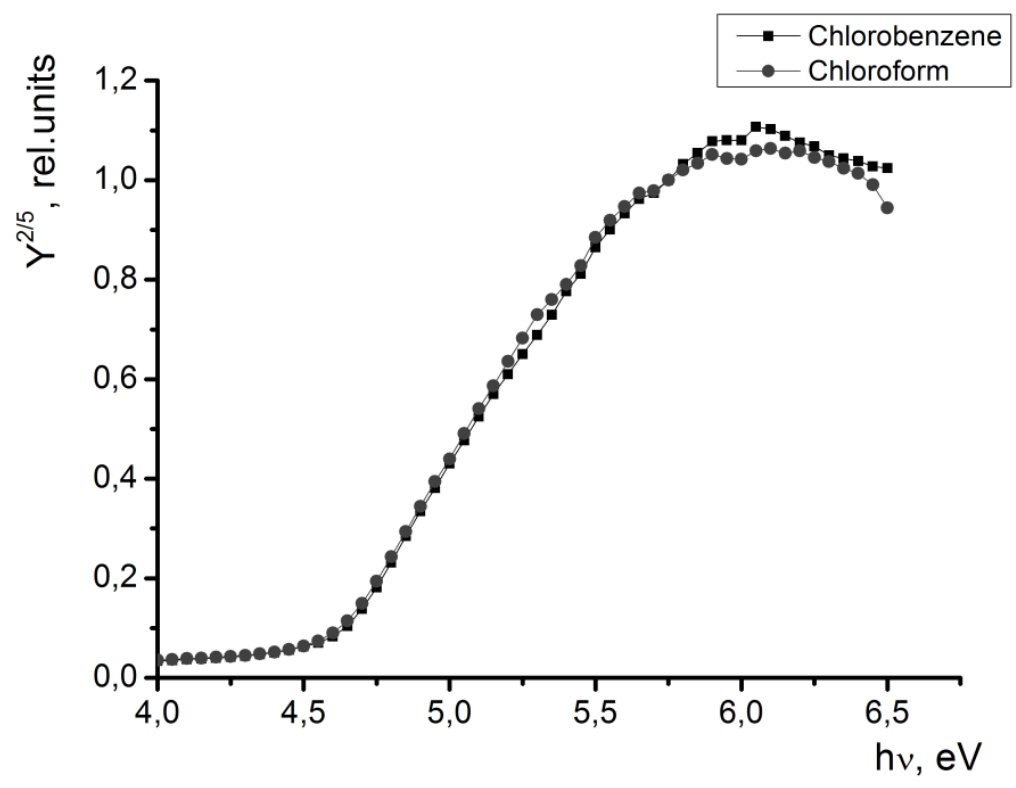

Fig.4 Photoemission yield spectra of P3HT thin films made of chlorobenzene and chloroform solution

Photoemission yield spectra of P3HT:PCBM samples made from chloroform solution are shown in Figure 5. At the P3HT:PCBM mass ratio of 1:1, the ionization energy of P3HT still remained the same as in the case of a bulky P3HT layer: $\mathrm{I}_{\mathrm{P} 3 \mathrm{HT}}=4.54 \pm 0.03 \mathrm{eV}$. Our obtained bulky layer ionization energy level values are in good agreement with the values obtained by UPS. While we have obtained values of $4.54 \pm 0.03 \mathrm{eV}$ for P3HT, Tsoi et al. report values of $4.60 \mathrm{eV}$ [22]. P3HT molecule ionization energy level shifts to a higher value when PCBM molecule concentration increases (see Fig.5).When the P3HT:PCBM mass ratio was 1:50, the ionization energy of P3HT had shifted by more than 
$0.40 \mathrm{eV}$ to the higher energy and reached $\mathrm{I}_{\mathrm{P} 3 \mathrm{HT}}=4.96 \pm 0.03 \mathrm{eV}$. It means that at the interface there is a higher difference between ionization energy of P3HT and electron affinity energy of PCBM than just comparing bulk values. P3HT ionization energy dependence on P3HT:PCBM mass ratio is shown in the inset of Fig.5. Energy shift could be attributed to either interface dipole or Fermi level alignment. It has been reported that interface dipole between P3HT and PCBM can create vacuum level shift of around $0.50 \mathrm{eV}[23,48]$. While it is in good agreement with our obtained energy shift value of $0.40 \mathrm{eV}$ still it cannot explain continuous threshold energy shift depending on P3HT:PCBM mass ratio. It has been reported that already $0.5 \mathrm{~nm}$ thick film is enough to reach maximum vacuum level shift at metal/organic interface in PYS measurements [26]. If the situation is similar in the case of OC-OC interface, P3HT:PCBM mass ratio of 1:3 or 1:5 should be enough to observe constant vacuum level shift. Further increase in the PCBM concentration should only increase the number of PCBM molecules close to the interface, instead of increasing interface dipole. On the other hand, the

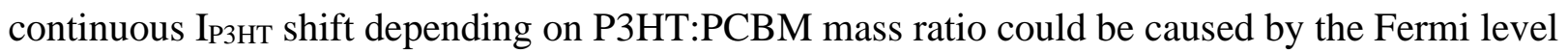
alignment at the OC-OC interface. When the PCBM concentration in the film increases, the number of P3HT molecules close to the OC-OC interface increases. It would similar to the planar heterojunction where the thickness of P3HT layer would decrease. The closer molecule is to the interface the more the energy levels will shift due to the alignment of Fermi levels. As the distance from the interface increases obtained ionization energy will be closer to the value of the bulk material. 


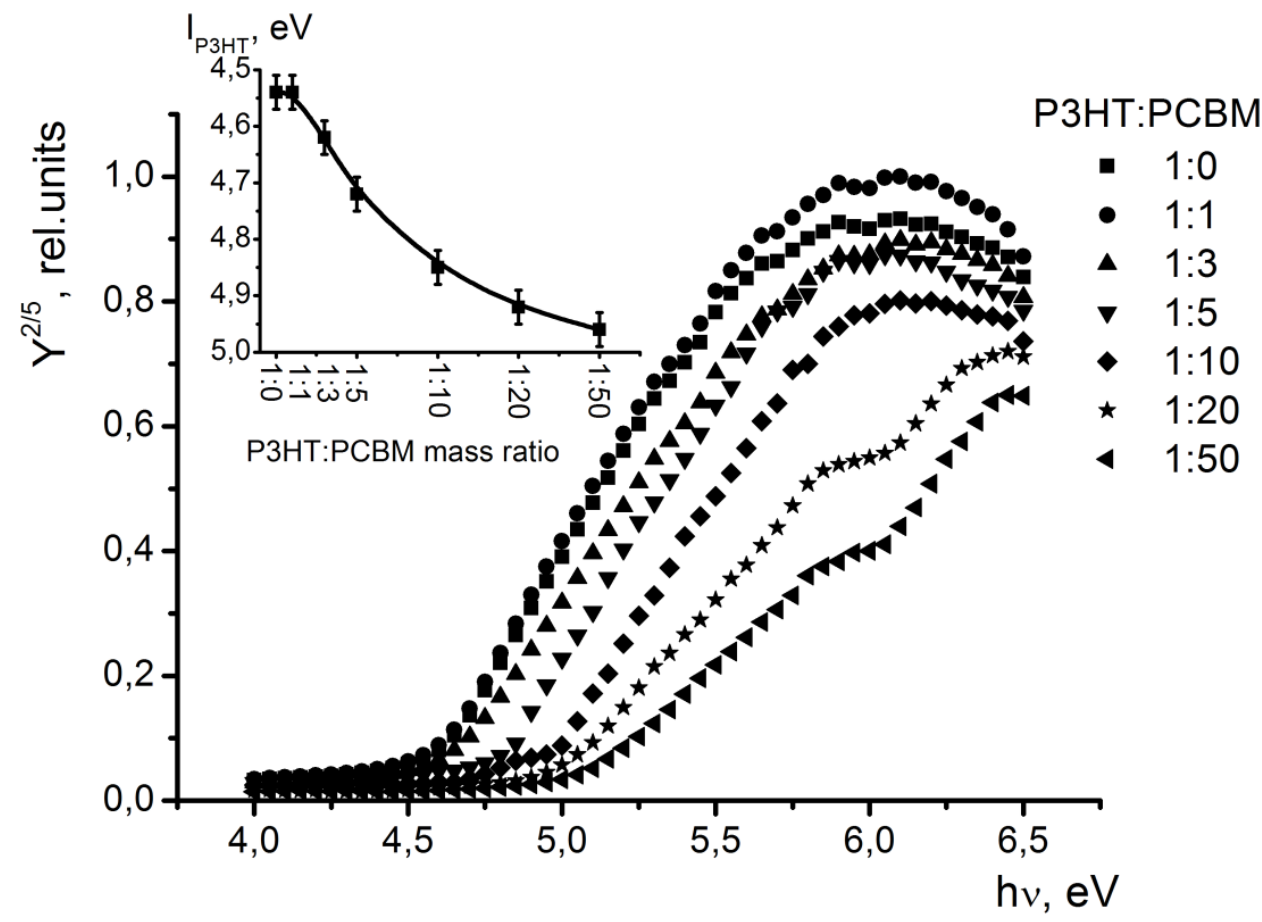

Fig.5 Photoemission yield spectra of bulk heterojunction samples depending on P3HT:PCBM mass ratio. Samples made from chloroform solution. Inset: P3HT ionization energy depending on P3HT:PCBM mass ratio

Signal decrease at higher PCBM concentration is related to the decreased number of P3HT molecules in the scanned layer. Only when the mass ratio of P3HT and PCBM was 1:20 and 1:50 signal coming from PCBM could be observed. In these cases the measured spectrum is a sum of two separate spectra [47]:

$$
Y(h v)=x\left(h v-I_{P 3 H T}\right)^{5 / 2} H\left(h v-I_{P 3 H T}\right)+y\left(h v-I_{P C B M}\right)^{5 / 2} H\left(h v-I_{P C B M}\right)
$$

where $\mathrm{I}_{\mathrm{P} 3 \mathrm{HT}}$ and $\mathrm{I}_{\mathrm{PCBM}}$ is ionization energy of P3HT and PCBM, respectively, $x$ and $y$ are constants showing relative signal intensity coming from each of the compounds, $H(E)$ is Heaviside step function. In both of these cases, the obtained ionization energy of PCBM was the same: $\mathrm{I}_{\mathrm{PCBM}}=6.15 \pm 0.03 \mathrm{eV}$. This value is in good agreement with our previously reported value for thin (below 12nm) PCBM layer on top of the P3HT film [32]. It means that despite the high concentration of PCBM, electrons are obtained from PCBM molecules that are close to P3HT molecules. 


\subsubsection{UPS measurements for bulk heterojunction samples made from chloroform}

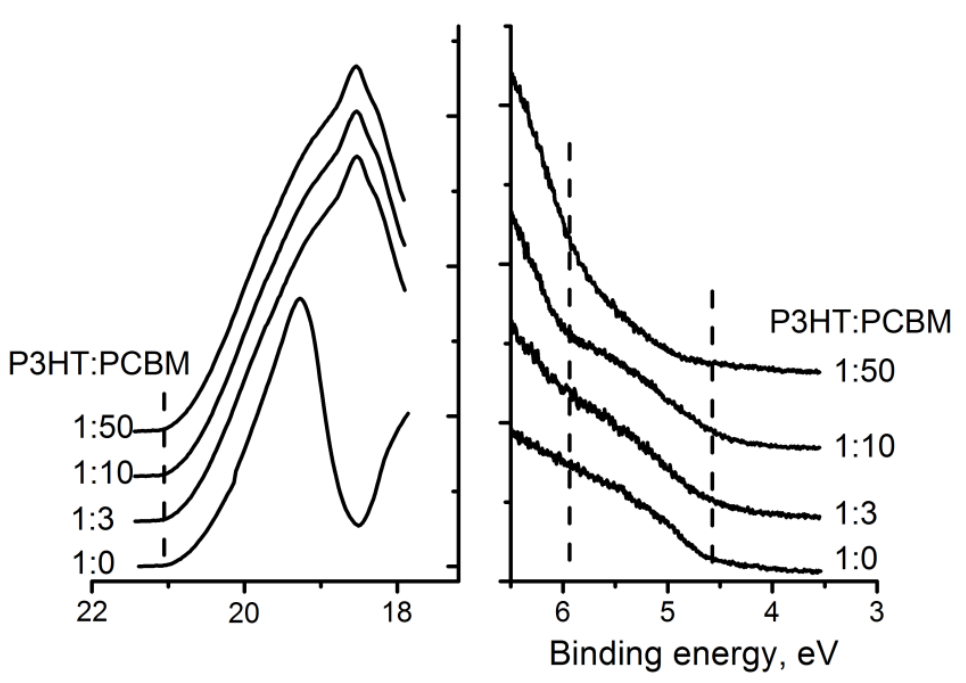

Fig.6 UPS spectra depending on P3HT:PCBM mass ratio showing secondary electron cutoff and low binding energy onset. Energy scale is shown with the respect to the P3HT vacuum level

The same samples made from chloroform were investigated by UPS method to compare both photoemission methods- UPS and PYS. Obtained ionization energy for P3HT in these measurements is $\mathrm{I}_{\mathrm{P} 3 \mathrm{HT}, \mathrm{UPS}}=4.6 \pm 0.1 \mathrm{eV}$ and does not depend on P3HT:PCBM mass ratio (see Fig.6). This value is in good agreement with the ionization energy value of thin P3HT films obtained using PYS (IP3HT= $4.54 \mathrm{eV}$ ). As it can be seen in Figure 6, for the samples where the P3HT:PCBM mass ratio was 1:3 to 1:50 we can observe increasing signal created by PCBM molecules. At the same time, P3HT signal is clearly diminished. Here the ionization energy of PCBM is IPCBM,UPS $=5.9 \pm 0.1 \mathrm{eV}$. This value is close to the previously reported value of IPCBM,UPS $=5.80 \mathrm{eV}[22,23]$. The obtained ionization energy value of $\mathrm{P} 3 \mathrm{HT}$ and $\mathrm{PCBM}$ correspond to the values of bulky materials instead of molecules at the OC-OC interface. This could be due to the low scanning depth of UPS combined with less homogeneously distributed compounds at the surface of the sample. UPS spectra of the samples with P3HT:PCBM mass ratio of 1:3, 1:10 and 1:50 are almost identical close to the secondary electron cutoff. Although there is a clear difference in the spectra between pristine P3HT sample and P3HT:PCBM samples, no secondary electron cutoff energy ( E $\left._{S E C O}\right)$ shift between pure P3HT sample and P3HT:PCBM samples was observed. The shift of the secondary electron cutoff (high binding energy cutoff) is considered to be related to the vacuum level shift, caused by the interface dipole. In 
our experiment (Fig.6) it cannot be observed. It fits the results obtained by PYS, where we concluded that the observed energy level shift could be related to the Fermi level alignment rather than vacuum level shift.

\section{CONCLUSIONS}

We have shown that the photoemission yield spectroscopy is a suitable method for organic compound- organic compound interface studies using bulk heterojunction samples. An important condition for the successful experiment is a poor phase separation of organic compounds within the bulk of the sample. It ensures that dominant signal is created by the OC-OC interface.

Our experiment showed that by lowering the portion of P3HT in the sample, the ionization energy of polymer shifted towards the ionization energy value of PCBM from $4.54 \mathrm{eV}$ to $4.96 \mathrm{eV}$. This could be mostly related to the alignment of Fermi levels of P3HT and PCBM.

In the UPS measurements, the samples made from chloroform solution showed energy level shift neither for P3HT nor for PCBM. The obtained ionization energy of P3HT and PCBM was similar to the value of bulk materials.

As it was shown, both of methods (UPS and PYS) had the same problem- PCBM could not be observed until extreme cases where the fullerene derivative mass portion in the sample was over $80 \%$. Further research concerning signal amplitude created by each compound should be done.

\section{ACKNOWLEDGEMENTS}

Financial support provided by Scientific Research Project for Students and Young Researchers Nr. SJZ2015/20 realized at the Institute of Solid State Physics, University of Latvia is greatly acknowledged.

Jennifer Mann from Physical Electronics is greatly acknowledged for providing UPS data. 


\section{REFERENCES}

1. To CH, Wong FL, Lee CS, Zapien J a. (2013) Transmission optimization of multilayer OLED encapsulation based on spectroscopic ellipsometry. Thin Solid Films 549:22-29. doi: 10.1016/j.tsf.2013.07.018

2. Cho AR, Kim EH, Park SY, Park LS (2014) Flexible OLED encapsulated with a gas barrier film and adhesive gasket. Synth Met 193:77-80. doi: 10.1016/j.synthmet.2014.03.027

3. Lee J, Yoshikawa S, Sagawa T (2014) Fabrication of efficient organic and hybrid solar cells by fine channel mist spray coating. Sol Energy Mater Sol Cells 127:111-121. doi:

10.1016/j.solmat.2014.04.010

4. Albrecht S, Grootoonk B, Neubert S, et al. (2014) Efficient hybrid inorganic/organic tandem solar cells with tailored recombination contacts. Sol Energy Mater Sol Cells 127:157-162. doi: 10.1016/j.solmat.2014.04.020

5. Wang H, Ji Z, Shang L, et al. (2011) Nonvolatile nano-crystal floating gate OFET memory with light assisted program. Org Electron 12:1236-1240. doi: 10.1016/j.orgel.2011.03.037

6. Cocchi M (2013) Organic Light-Emitting Diodes (OLEDs). Org Light diodes Mater devices Appl 293-318. doi: 10.1533/9780857098948.2.293

7. Xiao J, Liu X-K, Wang X-X, et al. (2014) Tailoring electronic structure of organic host for high-performance phosphorescent organic light-emitting diodes. Org Electron 15:2763-2768. doi: 10.1016/j.orgel.2014.08.006

8. Song S, Kim T, Bang SY, et al. (2013) Synthesis of the novel 2,2-bithiophene-3,3dicarboximide-based conjugated copolymers for OPVs. Synth Met 177:65-71. doi: 10.1016/j.synthmet.2013.06.012

9. Fan C, Yang P, Wang X, et al. (2011) Synthesis and organic photovoltaic (OPV) properties of triphenylamine derivatives based on a hexafluorocyclopentene "core." Sol Energy Mater Sol Cells 95:992-1000. doi: 10.1016/j.solmat.2010.12.010

10. Song S, Ko S-J, Shin H, et al. (2013) Pyrrolo[3,2-b]pyrrole based small molecules as donor materials for OPVs. Sol Energy Mater Sol Cells 112:120-126. doi:

10.1016/j.solmat.2013.01.004

11. Hayashi N, Ishii H, Ouchi Y, Seki K (2003) Examination of band bending at C60/metal interfaces by the Kelvin probe method. Synth Met 137:1377-1378. doi: 10.1016/S03796779(02)01149-9 
12. Ito E, Oji H, Hayashi N, et al. (2001) Electronic structures of TPD/metal interfaces studied by photoemission and Kelvin probe method. Appl Surf Sci 176:407-411. doi: 10.1016/S01694332(01)00088-5

13. Beerbom MM, Lägel B, Cascio a. J, et al. (2006) Direct comparison of photoemission spectroscopy and in situ Kelvin probe work function measurements on indium tin oxide films. J Electron Spectros Relat Phenomena 152:12-17. doi: 10.1016/j.elspec.2006.02.001

14. Gao Y (2010) Surface analytical studies of interfaces in organic semiconductor devices. Mater Sci Eng R Reports 68:39-87. doi: 10.1016/j.mser.2010.01.001

15. Tang JX, Tong SW, Lee CS, et al. (2003) Photoemission study of interface formation between ytterbium and tris-(8-hydroxyquinoline) aluminum. Chem Phys Lett 380:63-69. doi: 10.1016/j.cplett.2003.08.088

16. Schwieger T, Peisert H, Knupfer M (2004) Direct observation of interfacial charge transfer from silver to organic semiconductors. Chem Phys Lett 384:197-202. doi: 10.1016/j.cplett.2003.11.094

17. Park M, Woong J (2017) Anthracene-based perylene diimide electron-acceptor for fullerenefree organic solar cells. Dye Pigment 143:301-307. doi: 10.1016/j.dyepig.2017.04.057

18. Rybakiewicz R, Gawrys P, Tsikritzis D, et al. (2013) Electronic properties of semiconducting naphthalene bisimide derivatives - Ultraviolet photoelectron spectroscopy versus electrochemistry. Electrochim Acta 96:13-17. doi: 10.1016/j.electacta.2013.02.041

19. Salaneck WR (2009) Classical ultraviolet photoelectron spectroscopy of polymers. 174:3-9. doi: 10.1016/j.elspec.2009.03.024

20. Braun S, Salaneck WR, Fahlman M (2009) Energy-level alignment at organic/metal and organic/organic interfaces. Adv Mater 21:1450-1472. doi: 10.1002/adma.200802893

21. Xu Z, Chen L-M, Chen M-H, et al. (2009) Energy level alignment of poly(3-hexylthiophene): [6,6]-phenyl C[sub 61] butyric acid methyl ester bulk heterojunction. Appl Phys Lett 95:013301. doi: 10.1063/1.3163056

22. Tsoi WC, Spencer SJ, Yang L, et al. (2011) Effect of Crystallization on the Electronic Energy Levels and Thin Film Morphology of P3HT:PCBM Blends. Macromolecules 44:2944-2952. doi: $10.1021 / \mathrm{ma102841e}$

23. Guan Z-L, Kim JB, Wang H, et al. (2010) Direct determination of the electronic structure of the poly(3-hexylthiophene):phenyl-[6,6]-C61 butyric acid methyl ester blend. Org Electron 11:1779-1785. doi: 10.1016/j.orgel.2010.07.023 
24. El-Sayed A, Borghetti P, Goiri E, et al. (2013) Understanding Energy-Level Alignment in Donor À Acceptor / Metal Interfaces from Core-Level Shifts. ACS Nano 7:6914-6920. doi: $10.1021 / \mathrm{nn} 4020888$

25. Aygul U, Hintz H, Egelhaaf H, et al. (2013) Energy Level Alignment of a P3HT / Fullerene Blend during the Initial Steps of Degradation. J Phys Chem C 117:4992-4998. doi: $10.1021 / j p 4004642$

26. Kanai K, Honda M, Ishii H, et al. (2012) Interface electronic structure between the organic semiconductor film and electrode metal probed by photoelectron yield spectroscopy. Org Electron 13:309-319. doi: 10.1016/j.orgel.2011.11.024

27. Honda M, Kanai K, Komatsu K, et al. (2007) Atmospheric effect of air, N[sub 2], O[sub 2], and water vapor on the ionization energy of titanyl phthalocyanine thin film studied by photoemission yield spectroscopy. J Appl Phys 102:103704. doi: 10.1063/1.2809360

28. Grigalevicius S, Blazys G (2002) 3, 6-Di ( N-diphenylamino)-9-phenylcarbazole and its methyl-substituted derivative as novel hole-transporting amorphous molecular materials. Synth Met 128:127-131. doi: 10.1016/S0379-6779(01)00546-X

29. Seah M, Dench W (1979) Quantitative electron spectroscopy of surfaces: a standard database for electron inelastic mean free paths in solids. Surf Interface Anal 1:2-11. doi: 10.1002/sia.740010103

30. Monjushiro H, Watanabe I, Yokoyama Y (1991) Ultraviolet Photoelectron Yield Spectra of Thin Gold Films Measured in Air. Anal Sci 7:543-547. doi: 10.2116/analsci.7.543

31. Banoukepa GDR, Fujii A, Shimizu Y, Ozaki M (2015) 1,3,5-Tris(phenyl-2-benzimidazole)benzene cathode buffer layer thickness dependence in solution-processable organic solar cell based on 1,4,8,11,15,18,22,25-octahexylphthalocyanine. Jpn J Appl Phys 11:4-9. doi: 10.7567/JJAP.54.04DK11

32. Grzibovskis R, Vembris A (2016) Study of the P3HT/PCBM interface using photoemission yield spectroscopy. Proc SPIE 9895:98950Q. doi: 10.1117/12.2227823

33. Nam S, Shin M, Park S, et al. (2012) All-polymer solar cells with bulk heterojunction nanolayers of chemically doped electron-donating and electron-accepting polymers. Phys Chem Chem Phys 14:15046-15053. doi: 10.1039/c2cp43002a

34. Lu Y, Wang Y, Feng Z, et al. (2012) Temperature-dependent morphology evolution of P3HT:PCBM blend solar cells during annealing processes. Synth Met 162:2039-2046. doi: 10.1016/j.synthmet.2012.10.012 
35. Fukuda T, Toda A, Takahira K, et al. (2016) Molecular ordering of spin-coated and electrosprayed P3HT:PCBM thin films and their applications to photovoltaic cell. Thin Solid Films 612:373-380. doi: 10.1016/j.tsf.2016.06.019

36. Baek W-H, Yang H, Yoon T-S, et al. (2009) Effect of P3HT:PCBM concentration in solvent on performances of organic solar cells. Sol Energy Mater Sol Cells 93:1263-1267. doi: 10.1016/j.solmat.2009.01.019

37. Shen Y, Li K, Majumdar N, et al. (2011) Bulk and contact resistance in P3HT:PCBM heterojunction solar cells. Sol Energy Mater Sol Cells 95:2314-2317. doi: 10.1016/j.solmat.2011.03.046

38. Baek W-H, Yoon T-S, Lee HH, Kim Y-S (2010) Composition-dependent phase separation of P3HT:PCBM composites for high performance organic solar cells. Org Electron 11:933-937. doi: 10.1016/j.orgel.2010.02.013

39. Chen S, Zeng W, Su X, et al. (2015) Effect of preparation parameters on performance of P3HT: PCBM solar cells. Mater Sci Semicond Process 39:441-446. doi: 10.1016/j.mssp.2015.05.001

40. Müllerová J, Kaiser M, Nádaždy V, et al. (2016) Optical absorption study of P3HT:PCBM blend photo-oxidation for bulk heterojunction solar cells. Sol Energy 134:294-301. doi: 10.1016/j.solener.2016.05.009

41. Grzibovskis R, Vembris A, Pudzs K (2016) Relation between molecule ionization energy, film thickness and morphology of two indandione derivatives thin films. J Phys Chem Solids 95:12-18. doi: 10.1016/j.jpcs.2016.03.010

42. Xu Z, Chen L-M, Yang G, et al. (2009) Vertical Phase Separation in Poly(3-hexylthiophene): Fullerene Derivative Blends and its Advantage for Inverted Structure Solar Cells. Adv Funct Mater 19:1227-1234. doi: 10.1002/adfm.200801286

43. Swinnen a., Haeldermans I, van de Ven M, et al. (2006) Tuning the Dimensions of C60-Based Needlelike Crystals in Blended Thin Films. Adv Funct Mater 16:760-765. doi: 10.1002/adfm.200500812

44. Dang MT, Wantz G, Bejbouji H, et al. (2011) Polymeric solar cells based on P3HT:PCBM: Role of the casting solvent. Sol Energy Mater Sol Cells 95:3408-3418. doi: 10.1016/j.solmat.2011.07.039

45. Kane EO (1962) Theory of Photoelectric Emission from Semiconductors. Phys Rev 127:131141. 
46. Ow-Yang CW, Jia J, Aytun T, et al. (2014) Work function tuning of tin-doped indium oxide electrodes with solution-processed lithium fluoride. Thin Solid Films 559:58-63. doi: 10.1016/j.tsf.2013.11.035

47. Ozawa Y, Nakayama Y, Machida S, et al. (2014) Maximum probing depth of low-energy photoelectrons in an amorphous organic semiconductor film. J Electron Spectros Relat Phenomena 197:17-21. doi: 10.1016/j.elspec.2014.08.001

48. Aarnio H, Sehati P, Braun S, et al. (2011) Spontaneous Charge Transfer and Dipole Formation at the Interface Between P3HT and PCBM HOMO. Adv Energy Mater 792-797. doi: 10.1002/aenm.201100074

Institute of Solid State Physics, University of Latvia as the Center of Excellence has received funding from the European Union's Horizon 2020 Framework Programme H2020-WIDESPREAD-01-2016-2017TeamingPhase2 under grant agreement No. 739508, project CAMART ${ }^{2}$ 